\title{
MM HERCULIS: AN ECLIPSING BINARY OF THE RS CVn TYPE
}

\author{
J. R. SOWELL, * D. S. HALL, ** and G. W. HENRY***** \\ Dyer Observatory, Vanderbilt University, Nashville, Tenn. U.S.A. \\ E. W. BURKE, Jr.** \\ Department of Physics, King College, Bristol, Tenn., U.S.A. \\ and \\ E. F. MILONE** \\ Department of Physics, University of Calgary, Canada
}

(Received 27 September, 1982)

\begin{abstract}
Differential photoelectric photometry in $V, B$, and $U$ has been obtained for the eclipsing binary MM Her, a member of the RS Canum Venaticorum class. The light outside eclipse was Fourier-analyzed to study the wave. The migration and amplitude of the wave and the mean light level of the system now have been monitored from 1976 through 1980 . The phase of wave minimum has decreased from 0.80 to 0.25 , the wave amplitude has varied between $0^{m} .06$ and $0^{m} .12$, and the mean light level has fluctuated between 0.94 and 0.99 . Observations within eclipse revealed that the eclipses are partial, not total as previously thought, and a new time of mid-eclipse was found to be $\operatorname{JD}($ hel. $)=2444500.6665 \pm 0.0008$. The Russell model was used to solve the 1980 light curve. Elements of the rectified light curve were $i=86.35 \pm 0.09, r_{h}=0.070 \pm 0.002, r_{c}=0.125 \pm 0.001, L_{h}(V)=0.585 \pm 0.008$ and $L_{h}(B)=0.683 \pm 0.010$. The geometric elements were forced on the pre-1980 light curves and found to be compatible. With these elements and previously obtained double-lined radial velocity curves, new absolute dimensions have been calculated: $1.18 M_{\odot}$ and $1.58 R_{\odot}$ for the hotter star and $1.27 M_{\odot}$ and $2.83 R_{\odot}$ for the cooler star. By plotting color indices on the color-color curve, spectral types of G2V and K2IV were found, each uncertain by a couple of subclasses.
\end{abstract}

\section{Introduction}

MM Herculis is a member of a group of binaries that show a roughly sinusoidal light variation (wave) in their light curves outside eclipse. This group, named after RS Canum Venaticorum, a binary that typifies this peculiarity, consists of around fifty members. The original criteria for membership (Hall, 1976) include that the orbital period be between one and fourteen days, that the hotter star be a Main-Sequence or slightly evolved $\mathrm{F}$ or $\mathrm{G}$ star, and that $\mathrm{CaII} \mathrm{H}$ and $\mathrm{K}$ emission lines be seen outside the eclipses. Most of these binaries are unusual as intrinsically powerful radio and X-ray emitters. Walter et al. (1980) failed to detect MMHer with HEAO-I $\left(L_{x}<7.8 \times 10^{31} \mathrm{ergs} \mathrm{s}^{-1}\right)$

\footnotetext{
* Now at the Department of Astronomy, University of Michigan, Ann Arbor, Michigan.

** Guest Observer, Kitt Peak National Observatory, operated by the Association of Universities for Research in Astronomy, under contract with the National Science Foundation.

*** Now at McDonald Observatory, Fort Davis, Texas.
} 
but Walter and Bowyer (1981) did measure $L_{x}=5.8 \times 10^{30} \mathrm{ergs} \mathrm{s}^{-1}$ with HEAO-II. Spangler et al. (1977) failed to detect radio emission (upper limit $5 \mathrm{mJy}$ ). Another anomaly exhibited by many members is variability in the orbital period, but this phenomenon is not apparent in MMHer.

The variability in the light level is usually explained by the hypothesis that one of the two components, usually the cooler star, does not have a uniform distribution of brightness on its surface. An area of low surface brightness, a single large spot or a group of smaller spots, covers a significant fraction of one hemisphere. The spotted star rotates very nearly synchronously and consequently the wave remains approximately fixed in the light curve when brightness is plotted agains orbital phase. It is possible to remove the wave, and thereby correct the light curve in such a way that standard techniques can be used to solve for the geometrical elements.

Although several RS CVn-type binaries have been observed for almost a decade, most observations in such long series have been obtained only to monitor changes in the wave. MMHer has been observed photoelectrically for five consecutive years, but the observations have included data not only of the wave outside eclipse but also of the important eclipse phases. This combination makes it possible to solve the light curve and to derive photometric and geometric elements. Moreover, because double-lined radial velocity curves are available (Imbert, 1971; Popper, 1980, 1981), the masses and radii can be calculated.

Finally, because photometry has been obtained in three bandpasses, on the UBV system, a plot in the color-color diagram can provide estimates of the spectral types of the two stars.

\section{Photometric Data}

Differential photoelectric photometry was obtained by the observers listed in Table I; $n$ is the number of nights observed, $m$ is the total number of individual observations, and $\Delta t$ is the interval of time (in days) over which they were obtained. The telescopes

TABLE I

Tally of photoelectric photometry of MMHer

\begin{tabular}{|c|c|c|c|c|c|c|}
\hline Set & Observer & Observatory & mean JD & $\Delta t$ & $n$ & $m$ \\
\hline A & Burke & Kitt Peak & 2442955 & 18 & 13 & 45 \\
\hline B & Henry & Perkins & 2443021 & 98 & 21 & 69 \\
\hline $\mathrm{C}$ & Milone & Kitt Peak & 2443285 & 30 & 15 & 60 \\
\hline D & Burke & Kitt Peak & 2443298 & 14 & 9 & 40 \\
\hline $\mathrm{E}$ & Burke & Lowell & 2443320 & 12 & 5 & 18 \\
\hline $\mathrm{F}$ & Henry & Dyer & 2443354 & 224 & 36 & 120 \\
\hline G & Henry & Kitt Peak & 2443656 & 13 & 12 & 32 \\
\hline $\mathrm{H}$ & Henry & Kitt Peak & 2444095 & 137 & 16 & 46 \\
\hline I & Henry & Dyer & 2444188 & 8 & 2 & 7 \\
\hline $\mathbf{J}$ & Henry & Kitt Peak & 2444325 & 13 & 8 & 23 \\
\hline K & Sowell & Dyer & 2444460 & 49 & 16 & 296 \\
\hline $\mathbf{L}$ & Henry & Kitt Peak & 2444502 & 4 & 2 & 68 \\
\hline
\end{tabular}



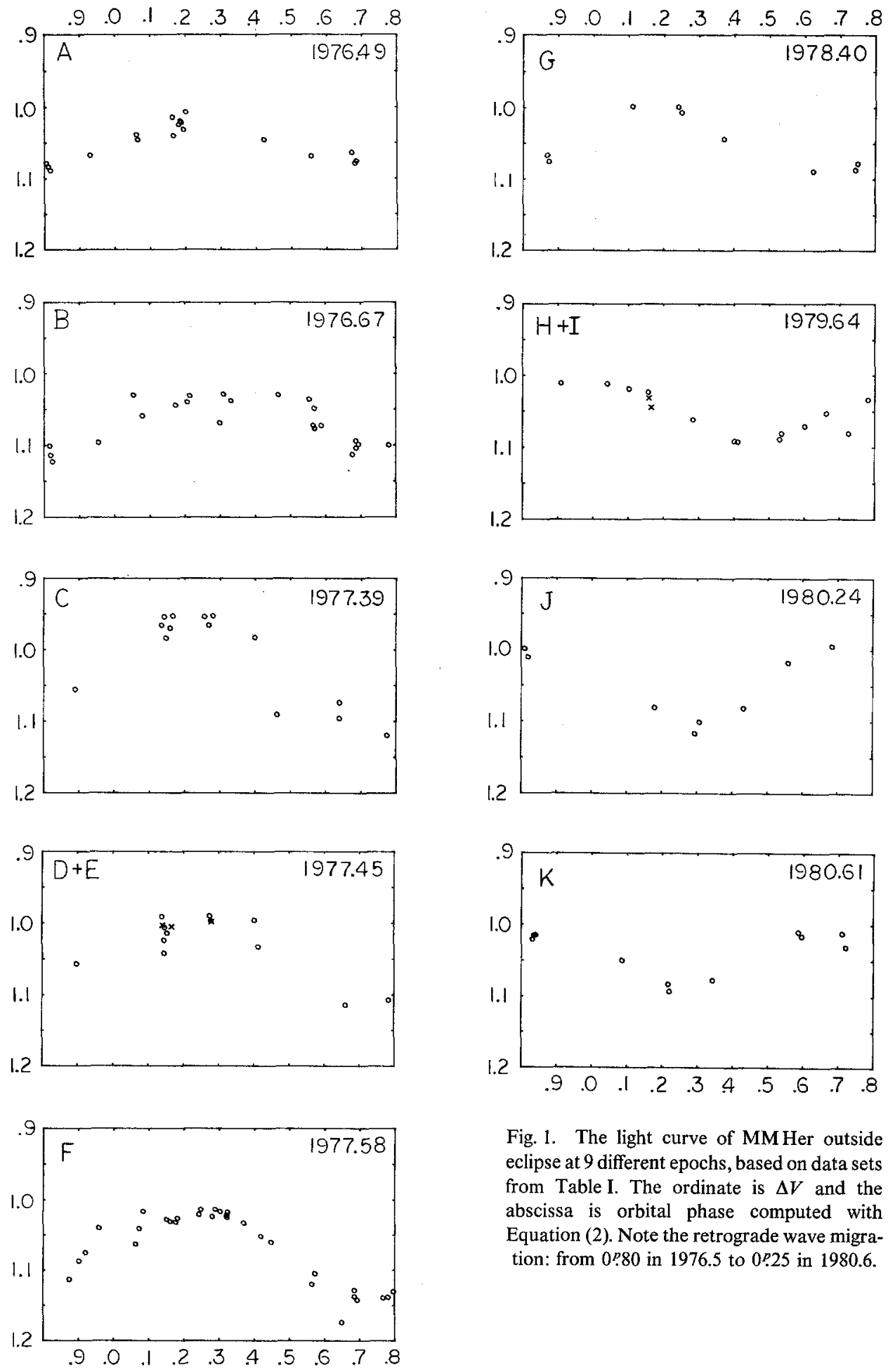

Fig. 1. The light curve of MMHer outside eclipse at 9 different epochs, based on data sets from Table $\mathrm{I}$. The ordinate is $\Delta V$ and the abscissa is orbital phase computed with Equation (2). Note the retrograde wave migration: from $0^{p} .80$ in 1976.5 to $0^{p} .25$ in 1980.6 . 
at Kitt Peak National Observatory were the No. 2 36-inch and the No. 4 16-inch; at Perkins Observatory, the 32-inch; at Lowell Observatory, the Morgan 24-inch; and at Dyer Observatory, the Seyfert 24-inch. The first four years contained few or no data within either eclipse. The last year, on the other hand, almost completely covered the primary eclipse, with the second set of observations by Henry in 1980 (set L) consisting of two nights of data entirely within eclipse.

Except for those of Milone, the differential measurements were made with $\mathrm{BD}+21^{\circ} 3274$ as the comparison star. During his first two years of observations, Henry used $\mathrm{BD}+22^{\circ} 3255$ as a check star. Milone, however, used $\mathrm{BD}+22^{\circ} 3256$ for the comparison star and $\mathrm{BD}+22^{\circ} 3259$ as the check star. A small diameter diaphragm was used consistently to exclude the light from the faint visual companion (Hall et al., 1977) about 18 arc sec away. Additional details concerning these observations are given by Henry (1979) and Sowell (1981).

The first three years of data were obtained in the visual $(V)$, blue $(B)$, and ultraviolet $(U)$ spectral regions, whereas the last two years did not include the $U$. The data were corrected for differential atmospheric extinction and transformed differentially to the standard $U B V$ photometric system. The atmospheric extinction coefficients used to reduce the data were previously determined seasonal averages, except for Milone's, which were averages determined during his intervals of observation. Heliocentric corrections were applied to the times of observation, which were taken approximately at the middle of each $V, B, U$, or $V, B$ sequence of deflections.

The 12 sets of data, numbered $A$ through $\mathrm{L}$ in correspondence with the listing in Table I, have been sent to the I.A.U. Commission 27 Archive of Unpublished Observations of Variable Stars (Breger, 1979), where they are available as file No. 110. Nightly averages of the outside-eclipse $V$ data from these sets of measurements are plotted in Figure 1. Sets D and E have been plotted together, as have sets $H$ and I. Set L, as we mentioned before, contained no data ouside eclipse.

\section{Time of Minimum}

Of all the available photoelectric data, only those of the night JD2444500 (set L) covered both branches of primary eclipse on the same night and, hence, seemed suitable for deriving a time of minimum. Before these data could be used for this purpose, the asymmetry of the eclipse branches due to the wave was removed with the equation

$$
l^{\text {cor }}=l^{\text {obs }}-\left(A_{1} \cos \theta+A_{2} \cos 2 \theta+B_{1} \sin \theta\right),
$$

where $A_{1}, A_{2}$, and $B_{1}$ are Fourier coefficients listed for set $\mathrm{K}$ in Table II. The phases were calculated with the ephemeris of Tsesevich (1954). A computer program was used to derive the time of mid-eclipse. It employs the technique of connecting two chronological points on one branch and measuring the horizontal distance between this line and a point on the opposite branch. The average of the midpoints of these lines is the adopted time of mideclipse. For both the visual and blue curves, this time occured at phase $0^{p} .9902 \pm 0.0001$, as determined from 17 and 14 midpoints, respectively. This 
TABLE II

Fourier coefficients for MMHer

\begin{tabular}{|c|c|c|c|c|c|c|c|}
\hline Set & $\lambda$ & $A_{0}$ & $A_{1}$ & $A_{2}$ & $B_{1}$ & $\Delta m$ & $\theta$ (min.) \\
\hline A & $V$ & $\begin{array}{r}0.9748 \\
\pm 0.0017\end{array}$ & $\begin{array}{r}0.0010 \\
\pm 0.0027\end{array}$ & $\begin{array}{l}-0.0033 \\
\pm 0.0025\end{array}$ & $\begin{array}{r}0.0278 \\
\pm 0.0018\end{array}$ & $\begin{array}{r}0.062 \\
\pm 0.004\end{array}$ & $\begin{array}{r}0^{P .74} \\
\pm 0^{p .02}\end{array}$ \\
\hline A & $B$ & $\begin{array}{r}0.9716 \\
\pm 0.0033\end{array}$ & $\begin{array}{r}0.0034 \\
\pm 0.0051\end{array}$ & $\begin{array}{l}-0.0074 \\
\pm 0.0048\end{array}$ & $\begin{array}{r}0.0269 \\
+0.0035\end{array}$ & $\begin{array}{r}0.061 \\
\pm 0.009\end{array}$ & $\begin{array}{r}0.73 \\
\pm 0.03\end{array}$ \\
\hline A & $U$ & $\begin{array}{r}0.9595 \\
\pm 0.0053\end{array}$ & $\begin{array}{l}-0.0032 \\
\pm 0.0083\end{array}$ & $\begin{array}{l}-0.0043 \\
\pm 0.0078\end{array}$ & $\begin{array}{r}0.0184 \\
\pm 0.0056\end{array}$ & $\begin{array}{r}0.042 \\
\pm 0.014\end{array}$ & $\begin{array}{r}0^{p} .78 \\
\pm 0^{p} .07\end{array}$ \\
\hline B & $V$ & $\begin{array}{r}0.9595 \\
\pm 0.0025\end{array}$ & $\begin{array}{l}-0.0093 \\
\pm 0.0038\end{array}$ & $\begin{array}{r}0.0051 \\
\pm 0.0034\end{array}$ & $\begin{array}{r}0.0315 \\
\pm 0.0028\end{array}$ & $\begin{array}{r}0.074 \\
\pm 0.007\end{array}$ & $\begin{array}{r}0^{p} .80 \\
\pm 0^{p} .02\end{array}$ \\
\hline B & $B$ & $\begin{array}{r}0.9625 \\
+0.0031\end{array}$ & $\begin{array}{l}-0.0070 \\
\pm 0.0047\end{array}$ & $\begin{array}{r}0.0072 \\
\pm 0.0042\end{array}$ & $\begin{array}{r}0.0327 \\
\pm 0.0035\end{array}$ & $\begin{array}{r}0.075 \\
+0.009\end{array}$ & $\begin{array}{r}0.78 \\
+0.02\end{array}$ \\
\hline B & $U$ & $\begin{array}{r}0.9533 \\
\pm 0.0101\end{array}$ & $\begin{array}{r}0.0268 \\
\pm 0.0163\end{array}$ & $\begin{array}{r}0.0234 \\
\pm 0.0138\end{array}$ & $\begin{array}{r}0.0342 \\
\pm 0.0067\end{array}$ & $\begin{array}{r}0.098 \\
\pm 0.025\end{array}$ & $\begin{array}{r}0.64 \\
\pm 0.05\end{array}$ \\
\hline C & $V$ & $\begin{array}{r}0.9427 \\
\pm 0.0054\end{array}$ & $\begin{array}{r}0.0159 \\
\pm 0.0078\end{array}$ & $\begin{array}{l}-0.0154 \\
\pm 0.0082\end{array}$ & $\begin{array}{r}0.0608 \\
\pm 0.0065\end{array}$ & $\begin{array}{r}0.145 \\
\pm 0.015\end{array}$ & $\begin{array}{r}0.71 \\
\pm 0.02\end{array}$ \\
\hline $\mathrm{C}$ & $B$ & $\begin{array}{r}0.9632 \\
\pm 0.0057\end{array}$ & $\begin{array}{r}0.0216 \\
\pm 0.0082\end{array}$ & $\begin{array}{l}-0.0073 \\
\pm 0.0086\end{array}$ & $\begin{array}{r}0.0527 \\
\pm 0.0068\end{array}$ & $\begin{array}{r}0.147 \\
\pm 0.016\end{array}$ & $\begin{array}{r}0^{p .76} \\
\pm 0^{p .02}\end{array}$ \\
\hline $\mathrm{C}$ & $U$ & $\begin{array}{r}0.9498 \\
\pm 0.0082\end{array}$ & $\begin{array}{l}-0.0033 \\
\pm 0.0123\end{array}$ & $\begin{array}{l}-0.0151 \\
\pm 0.0126\end{array}$ & $\begin{array}{r}0.0643 \\
\pm 0.0099\end{array}$ & $\begin{array}{r}0.147 \\
\pm 0.023\end{array}$ & $\begin{array}{r}0^{p} .76 \\
\pm 0^{p} .03\end{array}$ \\
\hline$D$ and $E$ & $V$ & $\begin{array}{r}0.9777 \\
\pm 0.0051\end{array}$ & $\begin{array}{r}0.0013 \\
\pm 0.0062\end{array}$ & $\begin{array}{r}0.0022 \\
\pm 0.0079\end{array}$ & $\begin{array}{r}0.0475 \\
\pm 0.0054\end{array}$ & $\begin{array}{r}0.107 \\
\pm 0.011\end{array}$ & $\begin{array}{r}0.75 \\
+0^{p} .02\end{array}$ \\
\hline $\mathrm{D}$ and $\mathrm{E}$ & $B$ & $\begin{array}{r}1.0046 \\
\pm 0.0054\end{array}$ & $\begin{array}{l}-0.0084 \\
\pm 0.0067\end{array}$ & $\begin{array}{r}0.0071 \\
\pm 0.0085\end{array}$ & $\begin{array}{r}0.0359 \\
\pm 0.0058\end{array}$ & $\begin{array}{r}0.080 \\
\pm 0.013\end{array}$ & $\begin{array}{r}0^{p .79} \\
\pm 0.03\end{array}$ \\
\hline$D$ and $E$ & $U$ & $\begin{array}{r}0.9968 \\
\pm 0.0056\end{array}$ & $\begin{array}{r}-0.0076 \\
\pm 0.0069\end{array}$ & $\begin{array}{r}0.0082 \\
\pm 0.0088\end{array}$ & $\begin{array}{r}0.0190 \\
\pm 0.0060\end{array}$ & $\begin{array}{r}0.044 \\
\pm 0.013\end{array}$ & $\begin{array}{r}0^{p .81} \\
+0^{p .05}\end{array}$ \\
\hline $\mathrm{F}$ & $V$ & $\begin{array}{r}0.9575 \\
\pm 0.0016\end{array}$ & $\begin{array}{r}0.0151 \\
\pm 0.0024\end{array}$ & $\begin{array}{r}0.0045 \\
\pm 0.0023\end{array}$ & $\begin{array}{r}0.0521 \\
\pm 0.0019\end{array}$ & $\begin{array}{r}0.122 \\
\pm 0.005\end{array}$ & $\begin{array}{r}0^{p} .705 \\
\pm 0^{p} .007\end{array}$ \\
\hline $\mathrm{F}$ & $B$ & $\begin{array}{r}0.9761 \\
\pm 0.0017\end{array}$ & $\begin{array}{r}0.0068 \\
\pm 0.0026\end{array}$ & $\begin{array}{r}0.0026 \\
\pm 0.0030\end{array}$ & $\begin{array}{r}0.0424 \\
\pm 0.0023\end{array}$ & $\begin{array}{r}0.096 \\
\pm 0.004\end{array}$ & $\begin{array}{r}0.72 \\
\pm 0.01\end{array}$ \\
\hline $\mathrm{F}$ & $U$ & $\begin{array}{r}0.9501 \\
\pm 0.0040\end{array}$ & $\begin{array}{l}-0.0069 \\
\pm 0.0062\end{array}$ & $\begin{array}{l}-0.0019 \\
\pm 0.0070\end{array}$ & $\begin{array}{r}0.0190 \\
\pm 0.0055\end{array}$ & $\begin{array}{r}0.046 \\
\pm 0.014\end{array}$ & $\begin{array}{r}0.80 \\
+0.05\end{array}$ \\
\hline G & $V$ & $\begin{array}{r}0.9769 \\
\pm 0.0024\end{array}$ & $\begin{array}{r}0.0209 \\
\pm 0.0033\end{array}$ & $\begin{array}{l}-0.0054 \\
\pm 0.0034\end{array}$ & $\begin{array}{r}0.0350 \\
\pm 0.0020\end{array}$ & $\begin{array}{r}0.091 \\
\pm 0.005\end{array}$ & $\begin{array}{r}0.66 \\
\pm 0.01\end{array}$ \\
\hline G & $B$ & $\begin{array}{r}0.9907 \\
\pm 0.0031\end{array}$ & $\begin{array}{r}0.0196 \\
\pm 0.0044\end{array}$ & $\begin{array}{l}-0.0079 \\
\pm 0.0045\end{array}$ & $\begin{array}{r}0.0279 \\
+0.0027\end{array}$ & $\begin{array}{r}0.075 \\
+0.007\end{array}$ & $\begin{array}{r}0.65 \\
\pm 0.02\end{array}$ \\
\hline G & $U$ & $\begin{array}{r}0.9682 \\
\pm 0.0031\end{array}$ & $\begin{array}{r}0.0155 \\
+0.0043\end{array}$ & $\begin{array}{l}-0.0107 \\
\pm 0.0045\end{array}$ & $\begin{array}{r}0.0198 \\
\pm 0.0026\end{array}$ & $\begin{array}{r}0.056 \\
+0.008\end{array}$ & $\begin{array}{r}0 ? .64 \\
\pm 0.02\end{array}$ \\
\hline $\mathrm{H}$ and $\mathrm{I}$ & $V$ & $\begin{array}{r}0.9770 \\
\pm 0.0037\end{array}$ & $\begin{array}{r}0.0360 \\
\pm 0.0056\end{array}$ & $\begin{array}{r}0.0064 \\
\pm 0.0068\end{array}$ & $\begin{array}{l}-0.0084 \\
\pm 0.0046\end{array}$ & $\begin{array}{r}0.082 \\
\pm 0.012\end{array}$ & $\begin{array}{r}0^{p} .46 \\
+0.02\end{array}$ \\
\hline $\mathrm{H}$ and $\mathrm{I}$ & $B$ & $\begin{array}{r}0.9924 \\
\pm 0.0040\end{array}$ & $\begin{array}{r}0.0340 \\
\pm 0.0059\end{array}$ & $\begin{array}{r}0.0014 \\
\pm 0.0073\end{array}$ & $\begin{array}{l}-0.0015 \\
\pm 0.0049\end{array}$ & $\begin{array}{r}0.074 \\
\pm 0.013\end{array}$ & $\begin{array}{r}0.49 \\
\pm 0.02\end{array}$ \\
\hline
\end{tabular}


Table II (continued)

\begin{tabular}{|c|c|c|c|c|c|c|c|}
\hline Set & $\lambda$ & $A_{0}$ & $A_{1}$ & $A_{2}$ & $B_{1}$ & $\Delta m$ & $\theta$ (min.) \\
\hline $\mathbf{J}$ & $V$ & $\begin{array}{r}0.9844 \\
+0.0078\end{array}$ & $\begin{array}{r}0.0082 \\
\pm 0.0131\end{array}$ & $\begin{array}{r}0.0103 \\
+0.0105\end{array}$ & $\begin{array}{l}-0.0483 \\
\pm 0.0052\end{array}$ & $\begin{array}{r}0.108 \\
\pm 0.012\end{array}$ & $\begin{array}{r}0.28 \\
\pm 0^{p} .04\end{array}$ \\
\hline $\mathrm{J}$ & $B$ & $\begin{array}{r}0.9910 \\
\pm 0.0058\end{array}$ & $\begin{array}{r}0.0027 \\
\pm 0.0098\end{array}$ & $\begin{array}{r}0.0039 \\
\pm 0.0079\end{array}$ & $\begin{array}{l}-0.0458 \\
\pm 0.0039\end{array}$ & $\begin{array}{r}0.100 \\
\pm 0.009\end{array}$ & $\begin{array}{r}0.26 \\
\pm 0.03\end{array}$ \\
\hline $\mathrm{K}$ & $V$ & $\begin{array}{r}0.9866 \\
\pm 0.0021\end{array}$ & $\begin{array}{l}-0.0016 \\
\pm 0.0032\end{array}$ & $\begin{array}{r}0.0146 \\
\pm 0.0031\end{array}$ & $\begin{array}{l}-0.0313 \\
\pm 0.0021\end{array}$ & $\begin{array}{r}0.069 \\
\pm 0.005\end{array}$ & $\begin{array}{r}0^{p .24} \\
\pm 0^{p} .02\end{array}$ \\
\hline K & $B$ & $\begin{array}{r}1.0027 \\
\pm 0.0022\end{array}$ & $\begin{array}{r}0.0043 \\
\pm 0.0033\end{array}$ & $\begin{array}{r}0.0168 \\
\pm 0.0032\end{array}$ & $\begin{array}{l}-0.0220 \\
\pm 0.0022\end{array}$ & $\begin{array}{r}0.049 \\
\pm 0.005\end{array}$ & $\begin{array}{r}0.28 \\
+0^{p} .02\end{array}$ \\
\hline
\end{tabular}

corresponds to an $\mathrm{O}-\mathrm{C}$ residual of $-0^{p} .0098=-0.078$ and a time of minimum of $\mathrm{JD}($ hel. $)=2444500.6665 \pm 0.0008$. An improved ephemeris for MMHer is thus

$$
\mathrm{JD}(\text { hel. })=2444500.6665+7^{d} \cdot 96037 E \text {. }
$$

Although several other times of minimum have been presented by Hall and Kreiner (1980), only a few came from photoelectric data. Consequently, we made no attempt to improve the period of Tsesevich.

\section{Fourier Analysis}

The brightness variation seen in the light curves outside eclipse was Fourier-analyzed by the equation

$$
l^{\mathrm{obs}}=A_{0}+A_{1} \cos \theta+A_{2} \cos 2 \theta+B_{1} \sin \theta,
$$

where $\theta$ is the orbital phase and $A_{0}, A_{1}, A_{2}$, and $B_{1}$ are the Fourier coefficients. In doing this the data sets were kept separate or, in two cases, combined in pairs, as indicated in the first column of Table II. All data points were used, except for those within $0^{p} 03$ of the centers of the eclipses. Unit luminosity was defined by the magnitude differences

$$
\Delta V=1^{m} .025, \quad \Delta B=1^{m} \cdot 210, \quad \text { and } \quad \Delta U=1^{m} \cdot 340
$$

used previously by Henry (1979). For the Milone data, though, the values

$$
\Delta V=1^{m} .873, \quad \Delta B=1^{m} \cdot 655, \quad \text { and } \quad \Delta U=1^{m} \cdot 147
$$

were used because Milone used a different comparison star. Milone's reference magnitudes were determined by observing the two comparison stars differentially with respect to each other at Dyer Observatory, then adding the resulting magnitude differences to Henry's reference magnitude. The resulting Fourier coefficients for MMHer are presented in Table II along with the corresponding values of $\Delta m$ (full amplitude of the wave in magnitude units) and $\theta$ (min.) (position of wave minimum), equations for which are given by Henry (1979). 


\section{Rectification}

Because useful tables and techniques are available for solving the light curve of an eclipsing binary in which the two stars are spherical and uniform in surface brightness except for the effects of limb darkening (Russell and Merrill 1952), it is necessary to rectify an observed light curve, i.e., convert it into a light curve produced by two spherical, limb-darkened stars. To accomplish this, both the light and the phase of an observation have to be modified.

Values of $l^{\text {obs }}$ in each set of data were rectified by the equation

$$
l^{\mathrm{rec}}=\frac{l^{\mathrm{obs}}+C_{0}+C_{1} \cos \theta+C_{2} \cos 2 \theta-(1-f)\left(A_{1}^{2}+B_{1}^{2}\right)^{1 / 2} \sin \left(\theta-\theta_{0}\right)}{\left(A_{0}+C_{0}\right)+\left(A_{2}+C_{2}\right) \cos 2 \theta},
$$

where $A_{0}, A_{1}, A_{2}$, and $B_{1}$ are the Fourier coefficients appropriate for each set, $C_{0}, C_{1}$, and $C_{2}$ are rectification coefficients for the reflection effect, $\theta_{0}=\theta(\min )+.90^{\circ}$, and $f$ is the fraction of the spotted star's light which is eclipsed. This is just the standard rectification equation of the Russell model with an added term which makes the spotted star's surface uniformly bright.

In using $A_{1}$ as well as $B_{1}$ to determine the distortion wave, it has been assumed implicitly that no part of $A_{1}$ was produced by the differential reflection effect. Moreover, the coefficients $C_{0}, C_{1}$, and $C_{2}$, which are derived from $A_{1}$, have been assumed to be zero. Neither assumption is logically justified but, because the two stars are not very different in spectral type and are not very close together, they are acceptable approximations.

Since the quantity $f$ could not be calculated before the light curve solution had been found, it also was assumed to be zero. The consequence of this assumption happens not to be harmful, as is shown in the next section.

Because Table Il shows that the average of the $A_{2}$ coefficients is very small or even positive $\left(A_{2}(V)=+0.002, A_{2}(B)=+0.003\right.$, and $\left.A_{2}(U)=-0.001\right)$, the ellipticity effect is negligible and therefore $z$ has been set equal to zero. Consequently, the rectified phases are the same as the unrectified ones.

\section{Light Curve Solutions}

Because the combined 1980 data of MM Her (sets J, K, and L) were the most complete in the eclipse phases, they alone were used in the initial light curve solution. To check the results, the geometrical elements were forced on all the earlier light curves, with only the relative luminosities allowed to vary. This made possible a determination of $L_{h}$ and $L_{c}$ in $V, B$, and $U$ from these earlier light curves.

The 1980 visual light curve of primary eclipse showed that the eclipses are partial, not total as had been previously thought. There is no evidence of the 0.06 totality first claimed by Tsesevich (1954). It was fortunate that secondary eclipse also had been 
observed, especially since the depth of secondary is necessary information for the solution of the light curve of a binary with partial eclipses.

Solving the light curve of a binary which undergoes partial eclipses is more difficult than if the eclipses were total, because there is one more unknown, $\alpha_{0}$, the normalized loss of light at mid-eclipse. The method we used was the one based on nomograms and tables of $\chi$ functions (Russell and Merrill, 1952). The first step was to plot the folded, rectified luminosity of primary eclipse vs the rectified phase. The second step was to divide the luminosity into ten equal increments of $n$. From this the values of $l_{0}$ (the luminosity at the bottom of eclipse) in the $V$ and $B$ bands, respectively, were found to be

$$
l_{0}^{\mathrm{pri}}=0.434 \text { and } \quad l_{0}^{\mathrm{pri}}=0.333
$$

at primary minimum and

$$
l_{0}^{\text {sec }}=0.869 \text { and } l_{0}^{\text {sec }}=0.893
$$

at secondary minimum.

As was discussed in the previous section, the assumption that $f=0$ means that rectification was not done properly. Generally, this would affect the depth of secondary eclipse if the cooler star is spotted, as is probably the case. Fortunately, because the position of $\theta$ (min.) happened to be very near phase 0.25 during 1980 , rectification neither added nor subtracted any light at phases $0^{p} .00$ and $0^{p .50}$. Consequently, because of this fortunate occurrence, the values of $l_{0}^{\mathrm{sec}}$ are correct afterall.

The nomogram solution process needed the value of $\chi(n=0.8)$ for primary eclipse, which was 0.389 for the $V$ and 0.383 for the $B$. On the nomograms the light curve solution is given by a point. The $\chi(n=0.8)$ values restricts the possibilities to a curve, but there are still an infinite number of points (solutions) on this curve. The possibilities are narrowed further by using the so-called depth line, which should intersect the $\chi(n=0.8)$ curve at a point. But there is a two-fold ambiguity depending upon whether the primary eclipse is an occultation or a transit. Since the system cannot be both, only one solution can be correct. Usually the other solution produces a poorer fit when the entire light curve is examined, (a) in the primary eclipse at phases other than those where $n=0.0, n=0.5$, and $n=0.8$ and (b) throughout secondary eclipse.

Nomograms designed for $x_{h}=x_{c}=0.6$ and $x_{h}=x_{c}=0.8$ were used for the visual light curve, but only the $x_{h}=x_{c}=0.8$ nomogram was used for the blue light curve. In all three cases no intersection between the calculated depth lines and the $\chi(n=0.8)$ curves occurred for the transit possibility, thus removing the ambiguity in the easiest possible way. There were intersections for the occultation possibility, and these intersection points yielded three approximately equal sets of values for the ratio of the radii $(k)$ and the geometrical depth $\left(p_{0}\right)$, being 0.572 and -0.827 , respectively.

Further work on the solution of this light curve was made much easier by a computer program of Eaton (1980) which uses the spherical model of Russell and Merrill (1952) to determine the elements. This program generates a theoretical curve from specified parameters, compares it with the observed data (rectified luminosities and phases), and 
determines the sum of the squares of the residuals. The program was modified to vary the parameters $i, r_{h}, r_{c}, x_{h}, x_{c}$, and $L_{h}$ by specific increments. Because $x_{h}$ and $x_{c}$ can be assumed, they were held constant and only the other four elements were allowed to vary. Starting with the aforementioned values $k=0.572$ and $p_{0}=-0.827$, assuming limb-darkening coefficients appropriate for each star, and using the $\chi(n=0.0)$ table to fix the phase of external contact at $\theta_{e}=0.0282$, we computed the values

$$
\begin{array}{ll}
r_{h}=0.066, & L_{h}(V)=0.585, \\
r_{c}=0.120, & L_{h}(B)=0.700,
\end{array}
$$

and

$$
i=86^{\circ} 35
$$

These comprised the initial set of parameters used with the Eaton computer program.

There were more than 300 data points from the 1980 primary (five nights) and secondary (two nights) eclipses combined. More than 250 different combinations of the parameters were tested. The best set of elements which fit both the $V$ and the $B$ light curves, i.e., the set with the smallest sum of the squares of the residuals, was found to be

$$
\begin{array}{rrr}
r_{h}=0.070, & L_{h}(V)=0.585 \\
\pm 2 & \pm 8 \\
r_{c}=0.125, & L_{h}(B)=0.683 \\
\pm 1 & & \pm 10
\end{array}
$$

TABLE III

Comparison of luminosities of MM Her

\begin{tabular}{lrrr}
\hline Set & \multicolumn{1}{c}{$L_{h}(V)$} & \multicolumn{1}{c}{$L_{h}(B)$} & \multicolumn{1}{c}{$L_{h}(U)$} \\
\hline F & 0.590 & 0.665 & 0.760 \\
& \pm 0.003 & \pm 0.003 & \pm 0.003 \\
D & 0.595 & 0.675 & 0.780 \\
& \pm 0.003 & \pm 0.005 & \pm 0.003 \\
E & 0.575 & 0.640 & 0.790 \\
& \pm 0.008 & \pm 0.008 & \pm 0.023 \\
$\mathrm{C}$ & 0.575 & 0.683 & 0.780 \\
& \pm 0.003 & \pm 0.003 & \pm 0.003 \\
$\mathrm{G}$ & 0.580 & 0.668 & 0.780 \\
& \pm 0.003 & \pm 0.003 & \pm 0.003 \\
$\mathrm{H}$ & 0.590 & 0.680 & \\
& \pm 0.003 & \pm 0.005 & \\
K and L & 0.585 & 0.683 & \\
& \pm 0.002 & \pm 0.002 & \\
\hline
\end{tabular}




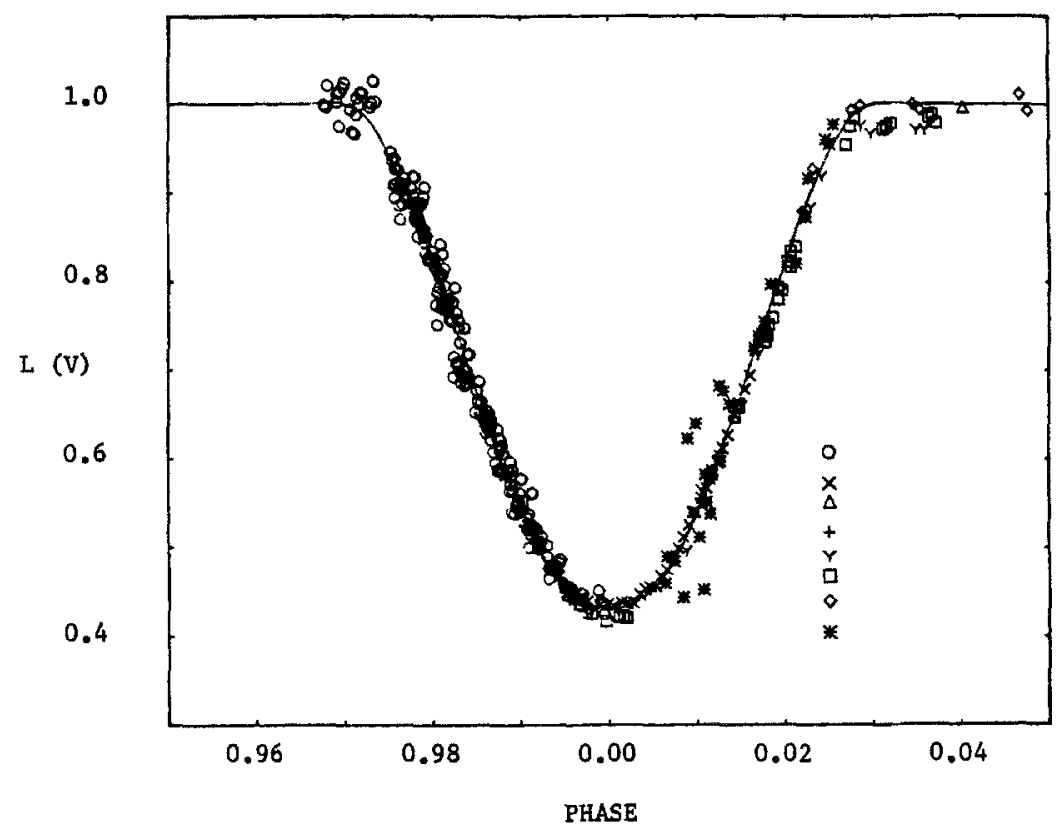

Fig. 2. MMHer in primary eclipse. The ordinate is rectified light in $V$. The curve represents the final set of elements. The symbols, in order from the top of the legend, refer to data in sets $\mathrm{K}, \mathrm{L}, \mathrm{H}, \mathrm{G}, \mathrm{D}, \mathrm{F}, \mathrm{E}$, and $\mathrm{C}$. The data from set $\mathrm{C}$ scatter because of very bad seeing on that night.

and

$$
\begin{array}{r}
i=86^{\circ} .35 \\
\pm \quad 9
\end{array}
$$

In this process of using many different combinations, the step sizes were as small as 0.05 in inclination, 0.005 in radii, and 0.0025 in luminosity. The errors assigned to each element were those which increased $\chi^{2}$ by one standard deviation.

These elements, except for the relative luminosities, were used with each set of pre-1980 eclipse data, to examine how well the geometrical elements fit these earlier data and to redetermine the value of $L_{h}$ from each set. Comparison of the 1980 values of $L_{h}$ with these other determinations of $L_{h}$, given in Table III, shows that all are consistent within estimated uncertainties. This technique of using the same geometrical elements made possible the determination of $L_{h}$ in $U$ from the earlier data, even though no ultraviolet observations had been obtained in 1980 .

Figure 2 shows the theoretical light curve in $V$ and the primary eclipse data from 1977 through 1980.

\section{Absolute Dimensions}

Besides being an eclipsing binary, MM Herculis is a double-lined spectroscopic binary. Imbert (1971) found $M_{h} \sin ^{3} i=1.22 \pm 0.07 M_{\odot}$ and $M_{c} \sin ^{3} i=1.19 \pm 0.06 M_{\odot}$, Popper (1980) reported $M_{h} \sin ^{3} i=1.2 M_{\odot}$ and $M_{c} \sin ^{3} i=1.25 M_{\odot}$, whereas Popper (1981) 
later revised those values slightly to be $M_{h} \sin ^{3} i=1.17 M_{\odot}$ and $M_{c} \sin ^{3} i=1.26 M_{\odot}$. The geometrical elements of the light curve solution we found in the previous section, along with the $M \sin ^{3} i$ values of Popper (1981), yield the absolute dimensions

$$
1.18 M_{\odot} \text { and } 1.58 R_{\odot}
$$

for the hotter star and

$$
1.27 M_{\odot} \text { and } 2.83 R_{\odot}
$$

for the cooler star. These radii, especially, should be more reliable than those given by either Imbert (1971) or Popper (1980), which they both noted as being uncertain.

\section{Spectral Types from Color Indices}

The various direct spectral classifications which have been published for the two components of MM Herculis are quite discordant. The Henry Draper Extension gives G0, apparently referring to the composite spectrum. Imbert (1971) gives G8 IV for the hotter primary and explains that he sees only the $\mathrm{H}$ and $\mathrm{K}$ emission lines of $\mathrm{Ca}$ II in the cooler secondary. Popper (1976) gives G2-5 for the hotter star and nothing for the cooler star. Later, Popper (1980) gives G2 for the hotter star and G8 for the cooler star but explains (Popper, 1981) that the G8 classification was uncertain.

The radii in the previous section suggest that the hotter star is a dwarf (approximate luminosity class $\mathrm{V}$ ) and that the cooler star is a subgiant (approximate luminosity class IV). Spectral types can be determined from color indices derived from solutions of light curves in three bandpasses. For this analysis we use the values of $L_{h}$ and $L_{c}$ corresponding to the 1978 light curve, because in that year observations were made in $U$ as well as in $B$ and $V$. For dwarfs and subgiants there are different relations connecting intrinsic color index $(B-V)_{0}$ with spectral type, but we resolve this ambiguity by assuming the hotter star is luminosity class $\mathrm{V}$ and the cooler star is luminosity class IV.

This technique was made possible for MM Her because Oliver (1974) had determined the apparent magnitudes of the comparison star $\mathrm{BD}+21^{\circ} 3274$. His values were

$$
\begin{aligned}
& V=8^{m} \cdot 46+0^{m} \cdot 03 \\
& B-V=0^{m} \cdot 64 \pm 0^{m} \cdot 02,
\end{aligned}
$$

and

$$
U-B=0^{m} \cdot 30 \pm 0^{m} \cdot 02 .
$$

The apparent magnitudes of MMHer were determined by adding the respective reference magnitudes and the $A_{0}$ Fourier coefficients (in magnitudes) to the comparison star's $V, B$, and $U$ values. This information, combined with the values of $L_{h}$ in Table III, yielded the magnitudes and color indices of each component given in Table IV. Popper (1981), though, gives slightly different values for the color indices of the combined light: 
TABLE IV

Color indices for $\mathrm{MM} \mathrm{Her}$

\begin{tabular}{|c|c|c|c|c|c|}
\hline & \multirow{2}{*}{$\frac{V}{\text { Oliver }}$} & \multicolumn{2}{|l|}{$B-V$} & \multicolumn{2}{|l|}{$U-B$} \\
\hline & & Oliver & Popper & Oliver & Popper \\
\hline Both stars & $9^{m} \cdot 51$ & $0^{m} \cdot 81$ & $0^{m} \cdot 84$ & $0^{m} \cdot 46$ & $0^{m} \cdot 39$ \\
\hline Hotter star & $10^{m} \cdot 10$ & $0^{m} \cdot 66$ & $0^{m} \cdot 69$ & $0^{m} \cdot 28$ & $0^{m} \cdot 22$ \\
\hline Cooler star & $10^{m} \cdot 45$ & $1^{m} \cdot 06$ & $1^{m} \cdot 10$ & $0^{m} \cdot 90$ & $0^{m} \cdot 84$ \\
\hline
\end{tabular}

$B-V=0^{m} .84$ and $U-B=0^{m} \cdot 39$. Color indices of each component derived from these values are given in Table IV also.

A plot of the color indices in a two-color diagram made us prefer the values based on Popper's photometry, because the other values made the hotter star fall below the locus of unreddened luminosity class $V$ stars. Even so, there was an ambiguity in interpreting Popper's values. This was because the hotter star lay on a portion of the locus which was very nearly parallel to the reddening trajectory. Therefore we explored two extreme possibilities and the intermediate case. (1) If $E(B-V)=0^{m} \cdot 00$, we get $(V-V)_{0}=0^{m} \cdot 69$ for the hotter star and $(B-V)_{0}=1^{m} \cdot 10$ for the cooler star, implying spectral types of G5V and $\mathrm{K} 2.5 \mathrm{IV}$, respectively, and an ultraviolet excess of $\delta(U-B)=0^{m} .13$ for the cooler star. (2) If $E(B-V)=0^{m} .12$, we get $(B-V)_{0}=0^{m} .57$ for the hotter star and $(B-V)_{0}=0^{m} .98$ for the cooler star, implying spectral types of F9V and K1 IV, respectively, and no ultraviolet excess for the cooler star. (3) In the intermediate case of $E(B-V)=0^{m} \cdot 06$, we get $(B-V)_{0}=0^{m} \cdot 63$ for the hotter star and $(B-V)_{0}=1^{m} .04$ for the cooler star, implying spectral types of G2V and K2 IV, respectively, and an ultraviolet excess of only $\delta(U-B)=0^{m} .04$ for the cooler star.

Compared to the classifications of Popper (1981), our intermediate case agrees perfectly for the hotter star but is about 4 spectral subclasses later for the cooler star.

Because the spectral type of the hotter star is similar to that of the Sun, it is easy to compute the approximate distance of MMHer. With its larger radius of $1.58 R_{\odot}$, it should have an absolute visual magnitude about $0^{m} .9$ brighter than the Sun, i.e., $M_{t}=3^{m} \cdot 7$. Using the apparent magnitude $V=10^{m} \cdot 10$ in Table IV, the distance would be 190 parsecs if $A_{v}=0^{m} .0$ or 210 parsecs if $A_{v}=0^{m} \cdot 2$. The value $E(B-V)=0^{m} \cdot 06$ corresponding to our intermediate case is consistent with interstellar absorption in the range $0^{m} 0<A_{V}<0^{m} .2$ and thus reasonable for a distance of around 200 parsecs.

\section{Distortion Wave Characteristics}

Figures 3, 4, and 5, respectively, show the variation with time of $\theta(\min ),. \Delta m$ in $V$, and $A_{0}$ in $V$. It is interesting to note in Figure 3 that, since 1976, $\theta$ (min.) has decreased smoothly from phase $0^{p} .80$ to 0.25 , with an apparent acceleration in the rate of decrease. The average rate corresponds to a migration period of $7.5 \mathrm{yr}$; in the context of a starspot model this corresponds to a rotation period $0.3 \%$ faster than synchronous. The 


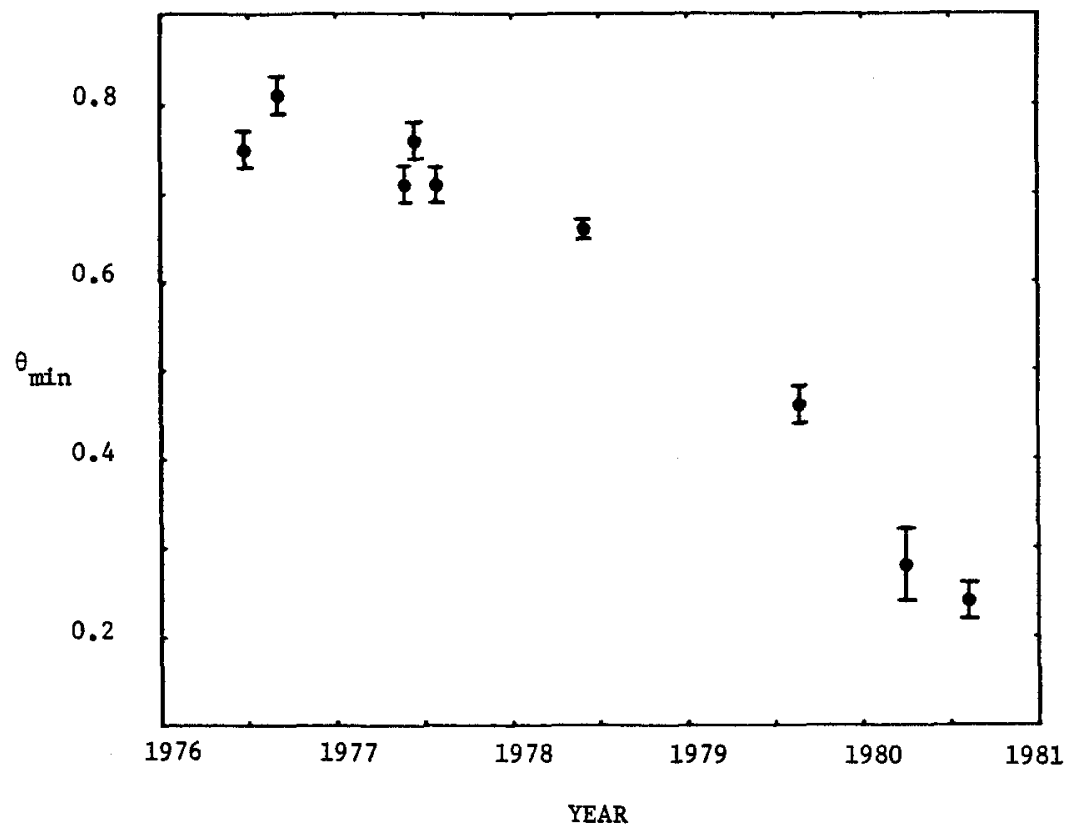

Fig. 3. The migration curve of MMHer. Each point is a mean of the $\theta$ (min.) values given in Table II for the different band passes.

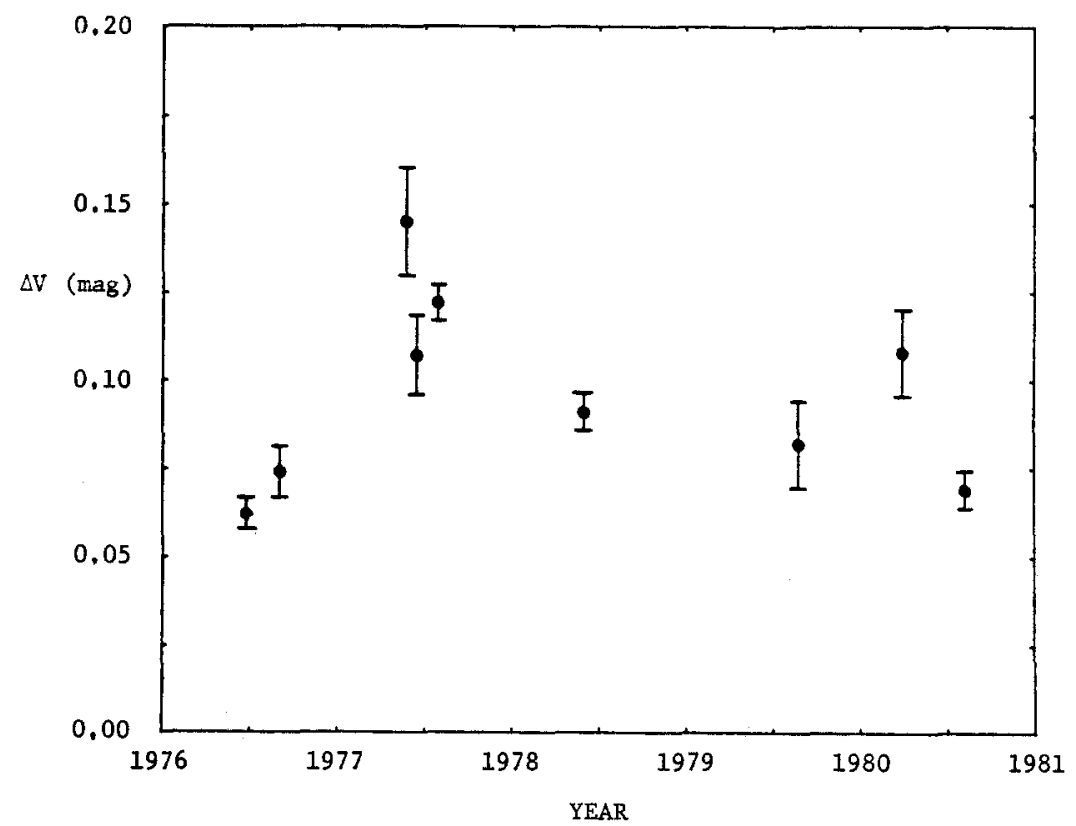

Fig. 4. The wave amplitude curve of MMHer in $V . \Delta V$ is the full amplitude of the wave in magnitudes, and the values are from Table II. 


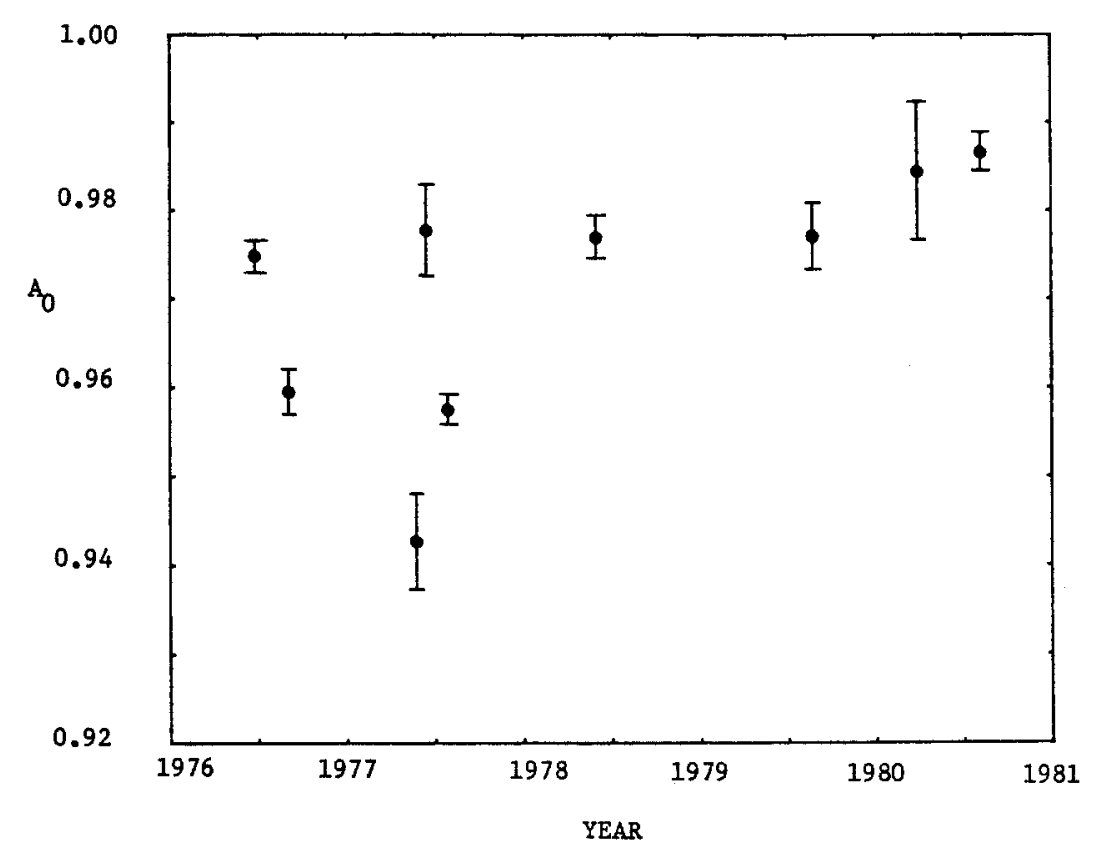

Fig. 5. The mean light level of MM Her outside eclipse in $V$. Values of $A_{0}$ for the $V$ light curves are from Table II.

smoothness of this curve indicates that the spot or spot group has maintained its identity over this five-year interval. The wave amplitude ( $\Delta m$ in $V$ ), on the other hand, has varied between $0^{m} .06$ and $0^{m} .12$ during this time and no pattern is recognizable in Figure 4. In Figure 5 it can be seen that the $A_{0}$ coefficients in $V$ show large fluctuations in 1976 and 1977 but in the last three years show a trend towards increasing value. The lowest value of $A_{0}$ is from Milone's data, but if our determination of the difference in magnitude between the two comparison stars is inaccurate, the $A_{0}$ value could possibly be higher. Monitoring of the wave in the light curve of MMHer will continue, increasing the baseline of data available for this well-observed RS CVn-type binary.

\section{Acknowledgements}

D.S.H. is happy to acknowledge that this work was supported in part by a grant (NSG-7543) from NASA. E.W.B. thanks Dr J. S. Hall for observing time at Lowell Observatory and Drs N. M. White and R. L. Millis for their assistance, and he expresses appreciation to the Research Corporation for support of his part of this research. E.F.M. is grateful for the support he received from the National Research Council of Canada, and thanks R. M. Robb, who assisted with his data reduction. 


\section{References}

Breger, M.: 1979, Inf. Bull. Var. Stars, No. 1659.

Eaton, J. A.: 1980 , private communication.

Hall, D. S.: 1976, in W. S. Fitch (ed.), "Multiple Periodic Variable Stars', Part I, IAU Colloq. 29, 287.

Hall, D. S. and Kreiner, J. M.: 1980, Acta Astron. 30, 387.

Hall, D. S., Henry, G. W., Burke, E. W., and Mullins, J. L.: 1977, Inf. Bull. Var. Stars, No. 1311.

Henry, G. W.: 1979, Master's thesis, Vanderbilt University, Nashville, Tennessee.

Imbert, M.: Astron. Astrophys. 12, 155.

Oliver, J. P.: 1974, Ph.D. thesis, University of California, Los Angeles.

Popper, D. M.: 1976, Inf. Bull. Var. Stars, No. 1083.

Popper, D. M.: 1980, Ann. Rev. Astron. Astrophys. 18, 115.

Popper, D. M.: 1981, private communication.

Russell, H. N. and Merrill, J. E.: 1952, Princeton Univ. Obs. Contr., No. 26.

Sowell, J. R.: 1981, Master's thesis, Vanderbilt University, Nashville, Tennessee.

Spangler, S. R., Owen, F. N., and Hulse, R. A.: 1977, Astron. J. 82, 989.

Tsesevich, V. P.: 1954, Odessa Izv. 4, Part 2, 116.

Walter, F. M. and Bowyer, C. S.: 1981, Astrophys. J. 245, 671.

Walter, F. M., Cash, W., Charles, P. A., and Bowyer, C. S.: 1980, Astrophys. J. 236, 212. 